\title{
ANALYSIS OF RELATIONSHIP BETWEEN BITCOIN EMISSION AND EXCHANGE RATES OF SELECTED FIAT CURRENCIES
}

\author{
Simona PAKĖNAITE் (1) ${ }^{1}$, Kamilè TAUJANSKAITE் (D) ${ }^{2}$ \\ ${ }^{l}$ Independent Researcher \\ ${ }^{2}$ Department of Financial Engineering, Faculty of Business Management, Vilnius Gediminas Technical University, \\ Sauletekio al. 11, Vilnius, Lithuania \\ ${ }^{*}$ E-mail: s.pakenaite@gmail.com
}

\begin{abstract}
Purpose - to investigate the possible influence of bitcoins and the Blockchain on exchange rates of selected fiat currencies by using quantitative analysis methods.

Research methodology - the importance, risks/opportunities are examined by studying scientific literature regarding cryptocurrencies and decentralization. The influence of bitcoins itself is examined by using regression analysis, ANOVA, descriptive statistics, correlation matrix, and VIF.

Findings - impart negative relation between Bitcoin money supply and exchange rates (USD/EUR) and a positive relation between Bitcoin inflation rate and exchange rates (USD/EUR). However, the actual influence is too negligible to make any significant affection for the conventional financial sector yet digitalization and decentralization are imminent.

Research limitations - only a few analyses have been conducted in a quantitative manner regarding cryptocurrencies impact. The influence of cryptocurrencies increased from 2011; phenomenon lacks long time data and examples to conduct an analysis model that applies to all assumptions without exceptions.

Practical implications - enterprises can embrace decentralization and digital money to keep up with the fast-growing market changes, improve transparency, and reduce costs. Close relation with fintech is indispensable; it promotes economic growth and accelerates the development of advanced operating practices.

Originality/Value - the interest in digital money and peer-to-peer payments constantly occupy greater importance of financial market and trading manner. Their influence is widely discussed but lacks quantitative researches based on actual historical data.
\end{abstract}

Keywords: cryptocurrency, Bitcoin, Blockchain, decentralization, banking, finances.

JEL Classification: C12, D53, F31, 031.

Conference topic: Contemporary Financial Management.

\section{Introduction}

Aspects of cryptocurrency and the Blockchain are globally discussed not only by investors but also by general society, policymakers, economists, and even national governments. There could be assumed that the effect on the society, public authorities, financial markets, and the banking sector is inevitable since the cryptocurrencies and its technology gained such popularity. It continually gains attention (positive or negative) from the media and the public. In recent years have emerged many types of cryptocurrencies (altcoins), such as Litecoin, Zcash, Dash, Ripple, Dogecoin, Gridcoin, Etherium, etc., nevertheless, the Bitcoin is primary and the most popular currency, with the highest market capitalization and price, and it is considered to have the supreme impact of all cryptocurrencies (Hileman \& Rauchs, 2017). However, there are many excitements about cryptocurrencies and the Blockchain. Optimists (e.g. Bollen, 2013; Luther \& White, 2014) believe that digital money and the Blockchain will alter payments and economics globally, however, pessimists (e.g. Shiller (Huillet, 2018); Carstens (Reid, 2018) considers Bitcoin to face a spectacular collapse near in the future. Hence, this analysis seeks to gather findings of the possible influence of nowadays technology trends for conventional financial manner.

Research object - bitcoins and decentralized technology. The main aim of this research is to investigate the presumptive influence of bitcoins on the traditional fiat currency market. The Bitcoin is assumed to represent all cryptocurrencies while studying the impact of factors related to the digital currency. This research implements a study of 

of selected fiat currencies

cryptocurrencies and the Blockchain technology by outlining main qualities, risks/opportunities, advantages/disadvantages. The key objectives are: to analyze different provisions of researchers based on their analyses regarding this topic; to analyze influence of cryptocurrency for currency markets in a quantitative way, by taking into consideration factors such fiat money exchange rates, bitcoin (BTC) market capitalization, money supply and inflation rate, cryptocurrencies transaction fees and price. The analysis consists of three main parts: (1) theoretical background of cryptocurrencies, (2) methodology of research method, as well as (3) practical analysis and results in interpretation. The first section covers an overview of cryptocurrencies and the Blockchain: pros/cons, technical considerations, main risks as well as limitations related to the topic, and etc. The second part describes methods of regression analysis: description, general considerations, assumptions and testing. The third unit represents the findings of the analysis and covers the interpretation of the outcome of quantitative investigations. The influence of bitcoins is examined based on actual historical data by using a regression analysis method. The regression analysis includes data of bitcoins such as market cap, price, transactions fees, inflation rate, and money supply of BTC and data of traditional financial activities (exchange rates USD/EUR) on a quantitative basis in the period of 2011-2018 (since the Bitcoin gained such popularity).

\section{Technical and economic background of cryptocurrencies and the Blockchain}

Currently, digital money is widely examined and discussed by economists, public authorities, such as European Bank Authority (since 2013), National banks (since 2014), Governments (2016), etc., as well as society itself. This innovation induces a lot of different opinions and attitudes based on various studies and researches as well as personal judgment. There are no long - time data series for analysis because the organized exchange of cryptocurrencies and fiat money started only in 2010. Since this topic is in a great request, many systematic types of research related with cryptocurrencies especially Bitcoin have been made (e.g. Nair \& Sebastian, 2017; Narayanan, Bonneau, Felten, Miller, \& Goldfeder, 2016; Zaninotto, 2016, etc.), however, only one analysis has been conducted in a quantitative manner regarding increasing influence.

In accordance, this part of the research provides the general concept about cryptocurrencies and the Blockchain (key opportunities, risks, benefits, simplified technical description, etc.), traditional money, and their economic and financial relationships by emphasizing specialists' opinions and concluding scientific investigations.

\subsection{Opportunities and risks of cryptocurrencies}

There are many substantial forces that constantly increase the demand for cryptocurrencies. These forces could be divided into economic and individual aspects (European Banking Authority [EBA], 2014), which bring potential benefits to the society and the country's wealth being. This classification is shown in Table 1.

Table 1. Economic and individual benefits of cryptocurrencies (created by an author based on EBA, 2014)

\section{ECONOMIC BENEFITS}

Transaction costs: without intermediaries, transactions with cryptocurrencies can currently be achieved at lower costs compared to the other payment tools, such as bank transfers; this is related with security which takes time for banks to verify transfers

Transfer fulfillment time: transactions with cryptocurrencies are executed faster than with fiat money. For example, with Bitcoin to verify and complete the transaction it takes from 10 to 60 minutes. Approximately, every new block in a chain is added every 10 minutes. Also, transfers are made on a $24 / 7$ basis

Contributing economic growth: cryptocurrencies provided new opportunities for enterprises as well as induced new types of business which did not exist before, such as developments of mining hardware, commercial mining services, etc.

Financial inclusion: scheme of virtual currency provides alternative ways to effective transactions, where users do not have access to cheap and convenient financial services as well as where the administrative barriers for obtaining an account is too high

\section{INDIVIDUAL BENEFITS}

Security of personal data: transfers with cryptocurrencies do not require to disclose personal or sensitive information elsewise than making bank payment, which requires password or credit card data. It reduces identity theft possibility

Limited intervention with public authorities: sometimes payments with fiat currencies are considered to be trustless because of banks and governments' ability to control

Moreover, systematically features of cryptocurrencies could be breakdown into transactional and monetary (Rosic, 2016). Transactional specialties are the following ones:

- Protected integrity: after the transaction has been confirmed it cannot be changed or duplicated.

- Anonymity: each transaction is confidential (does not require to include personal information).

- Permissiveness: nor individual neither organization is in a control of cryptocurrency (decentralization). 
- Fast and worldwide: transaction could be made everywhere; the physical location of the computer does not have an impact as well as the transaction is confirmed instantly in a network.

- Security: cryptocurrency funds are hidden under the public key cryptography system.

Monetary characteristics of cryptocurrencies are as per below:

- Controlled money supply: Almost every kind of cryptocurrencies restrains the supply of its units by a schedule written in its unique code. For example, only 21 million Bitcoins can be mined in total.

- No debt: The fiat money in the market is created by debt. Cryptocurrencies do not represent debts (Rosic, 2016).

- Convertibility: cryptocurrencies could be simply converted to fiat currency or exchanged to other virtual money.

Nevertheless, the European Banking Authority (EBA, 2014) emphasizes various hazards related to virtual money. They distribute risks into five key categories. Every main category has a subgroup where risks are indicated with the specific details as well as a rank level. In accordance with EBA, Table 2 presents the fundamental risks of cryptocurrencies.

Table 2. Risks of cryptocurrencies (created by an author based on EBA, 2014)

\begin{tabular}{|c|c|c|}
\hline KEY CATEGORY & DESCRIPTION & RANK \\
\hline \multirow{9}{*}{ (A) Risk for users } & User suffers the loss when an exchange is fraudulent & High \\
\hline & User holding $\mathrm{VCs}^{1}$ may unexpectedly become liable to tax requirements & Med \\
\hline & User suffers loss due to changes made to the VC protocol and other core components & High \\
\hline & User loses VC units through e-wallet theft or hacking & High \\
\hline & $\begin{array}{l}\text { Market participants suffer losses due to the unexpected application of the law that renders con- } \\
\text { tracts illegal/unenforceable }\end{array}$ & Med \\
\hline & $\begin{array}{l}\text { User suffers the loss when a counterparty fails to meet contractual payment or settlement obli- } \\
\text { gations }\end{array}$ & High \\
\hline & $\begin{array}{l}\text { The user has no guarantee that VCs are accepted by merchants as a means of payment on a } \\
\text { permanent basis }\end{array}$ & High \\
\hline & The user is not able to convert VCs to fiat currency, or not at a reasonable price & High \\
\hline & User suffers loss as a result of VC prices being manipulated & High \\
\hline \multirow[b]{2}{*}{$\begin{array}{l}\text { (B) Risks for non-user } \\
\text { market participants }\end{array}$} & After accepting VC for payment, the merchant is not reimbursed & High \\
\hline & $\begin{array}{l}\text { The exchange is operationally unable to fulfill payment obligations denominated in VCs or } \\
\text { FCs }\end{array}$ & High \\
\hline \multirow{2}{*}{$\begin{array}{l}\text { (C) Risks for financial } \\
\text { integrity }\end{array}$} & $\begin{array}{l}\text { Criminals are able to launder proceeds of crime because they can deposit/transfer VCs glob- } \\
\text { ally, rapidly and irrevocably }\end{array}$ & High \\
\hline & Criminal organizations can use VCs to settle internal or inter-organizational payments & Med \\
\hline $\begin{array}{l}\text { (D) Risks for payment } \\
\text { systems in FCs }\end{array}$ & $\begin{array}{l}\text { Businesses in the real economy suffer losses due to disruptions in financial markets that were } \\
\text { caused by VC assets blocked, delayed, etc. }\end{array}$ & Low \\
\hline \multirow{3}{*}{$\begin{array}{l}\text { (E) Risks for regula- } \\
\text { tory authorities }\end{array}$} & $\begin{array}{l}\text { Should VCs gain widespread acceptance, central bank as issuer of FC can no longer steer the } \\
\text { economy, as the impact of its monetary measures become difficult to predict }\end{array}$ & Low \\
\hline & $\begin{array}{l}\text { Regulation and supervision of conventional financial activities are circumvented by unregu- } \\
\text { lated "shadow" activities that incur the same risks }\end{array}$ & Med \\
\hline & $\begin{array}{l}\text { Regulators prevent potential new entrants to payment services market if the regulatory ap- } \\
\text { proach to VCs is excessive }\end{array}$ & Med \\
\hline
\end{tabular}

To summarize Table 2 above, cryptocurrencies could cause risks for users, non-users market participants, regulatory authorities, financial integrity as well as payment systems.

\subsection{Review of the Bitcoin}

The first cryptocurrency Bitcoin was created and introduced in 2009 by an anonymous person or a group of people called Nakamoto (2009). In accordance with Antonopoulos (2014), the Bitcoin is defined as the collection of concepts and technologies that form the basis of digital money ecosystem. Units of this currency are exploited to store and transmit value between participants in the network of Bitcoin. Users of the network communicate by using the BTC protocol, which is the available open source software called Blockchain. Therefore, the usage of cryptocurrencies

${ }^{1} \mathrm{VCs}-$ virtual currencies. 
accelerated after Bitcoin and its unique technology were presented (Zhao, 2015). By creating Bitcoin, Nakamoto solved many problems related to digital currencies at once, such as (1) avoiding duplication or (2) controlling the number of units in the market. The Bitcoin is used effectively, independently, and with the low-cost transfer of funds (Nakamoto, 2009).

Moreover, literature emphasizes some advantages and disadvantages of the Bitcoin. One of the biggest benefits of digital money is (1) decentralization, there is no need for an intermediary for the transactions to occur; (2) anonymous trades which ensures privacy of personal data; (3) fungibility of exchanging money; (4) some of the specialists consider it as an advantage that cryptocurrencies are nonpolitical, and government do not have any influence for its use (except the legal issues); (5) transparency is one more pro of the Bitcoin whereas its technology is open and everyone is available to see networks information. However, there are some disadvantages as well: (1) high price volatility since currency is a novelty and is based only on belief; (3) lack of knowledge in a society; (4) decentralization and anonymity cause legal and regulatory compliance as well as (5) money laundering and (6) expansion of "Black market". Hence, there are a lot of different prevailing attitudes on cryptocurrencies and Bitcoin is the most controversially discussed one (D. Tapscott \& A. Tapscott, 2016).

\subsection{Investigations of cryptocurrencies and its technology: findings of researchers}

There are plenty of opinions in nowadays society about the use of cryptocurrencies and the Blockchain. Optimists believe that digital money and the Blockchain will improve payment system and could be the alternative way for it. Pessimists suppose that Bitcoin is one more economic bubble (Table 3). Vejačka (2014) in his research analyzed basic features of cryptocurrencies. The research revealed that the volatility of cryptocurrencies exchange rates is way higher than basic indices. According to the author, this kind of volatility causes a greater risk of trading cryptocurrency due to the reflection in the formation of price bubbles. Nevertheless, the growing value of exchange rates attracts a lot of speculators, this led to an understanding of cryptocurrencies not as a payment medium, but rather as specific commodities for investment. Also, compared with other commodities, cryptocurrencies have the benefit of easy portability. The author summarized the basic aspects of cryptocurrencies, which is: volatility (standard deviation method and Average True Range analysis were used), awareness, availability, anonymity, and legislation influence.

Furthermore, in accordance with Bollen (2013), Bitcoin has a number of weaknesses mostly related to the longterm viability issues for the economic (especially high volatility). However, the author believes that virtual currencies can and probably will succeed in time, as innovators build on the lessons from the Bitcoin experience. Also, the Nobel prizewinning economist Shiller examined that the cryptocurrencies will not be a permanent feature of the financial world. He indicated Bitcoins as a very clever idea, but he is impressed more with the technology than cryptocurrencies itself. Economist thinks of cryptocurrencies more as an experiment (Wearden, 2018).

Interesting that Glaser, Kai, Haferkorn, Weber, and Siering (2014) indicated that the demand for exchanging digital money to domestic currency increases along with the attention on Bitcoin whether the actual use of it. Also, authors and literature emphasize that Bitcoin users rather use it as an asset than as a currency. New users of Bitcoin lead to the increased volume of currency traded than to the enhanced extent within the BTC system (Glaser et al., 2014).

Table 3. Different provisions of researchers (created by an author)

\begin{tabular}{|c|c|l|}
\hline AUTHOR & YEAR & \multicolumn{1}{|c|}{ FINDINGS } \\
\hline Robert Bollen & 2013 & $\begin{array}{l}\text { The author believes that virtual currencies can and probably will succeed in time, as in- } \\
\text { novators build on the lessons from the Bitcoin experience }\end{array}$ \\
\hline Martin Vejačka & 2014 & $\begin{array}{l}\text { Investigates cryptocurrencies not as a payment medium, but rather as specific com- } \\
\text { modities for investment }\end{array}$ \\
\hline $\begin{array}{c}\text { William J. Luther and } \\
\text { Lawrence H. White }\end{array}$ & 2014 & $\begin{array}{l}\text { The exchange pricing and instantaneous exchange facilities enable Bitcoin to function } \\
\text { as a mean of exchange. The evolution of virtual currencies provides the ability to re- } \\
\text { flect alternative payment system with non-state money }\end{array}$ \\
\hline F. Glaser et al. & 2014 & $\begin{array}{l}\text { New users of Bitcoin lead to the increased volume of currency traded than to the en- } \\
\text { hanced extent within the BTC system, which represents that cryptocurrencies currently } \\
\text { is more considered as an asset }\end{array}$ \\
\hline Alina Khvan & 2016 & $\begin{array}{l}\text { The author investigated that Bitcoin had a significant effect on the US dollar in the } \\
\text { 2014-2016 }\end{array}$ \\
\hline Robert Shiller & 2018 & $\begin{array}{l}\text { Professional specifies that cryptocurrencies will not be a permanent feature of the } \\
\text { financial world and he is more impressed the Blockchain (Huillet, 2018) }\end{array}$ \\
\hline Augustin Carstens & 2018 & $\begin{array}{l}\text { Author imparted Bitcoin as the economic bubble and that bitcoin is not suitable for } \\
\text { payments (Reid, 2018) }\end{array}$ \\
\hline
\end{tabular}

Also, according to Luther and White (2014), an inelastic supply very volatile demand makes the price of Bitcoin very unstable. However, they impart that this is not a huge drawback of cryptocurrencies to spread as the medium of 
exchange. The exchange pricing and instantaneous exchange facilities enable Bitcoin to function as a mean of exchange while distributing the speculative risk of holding it to those who are the most willing to bear it. Although, the evolution of Bitcoin and other virtual currency will continue to provide the opportunity to reflect alternative payment system with non-state money.

However, the influence of cryptocurrencies was already examined by one specialist. Khvan (2016) created a regression analysis to investigate the affection of cryptocurrencies for the US currency. The author indicated two relevant propositions: if the number of Bitcoins in its circulation increases by 1 million, EUR/USD rate rises. Therefore, finding proposes that the dissemination of cryptocurrency reduces US dollar. Moreover, the author confirmed a hypothesis, that Bitcoin had no significant effect on the US dollar in 2009-2010, but in the 2014-2016 (last period) cryptocurrency had significant affection. Moreover, in accordance with Houben and Snyers (2018), cryptocurrencies have skyrocketed in 2017, and currently, hundreds of coins are circulating in the market with a total market capitalization of over than EUR 300 billion. Numbers tend to increase on a regular basis, therefore, impact on fiat currency markets will increase as well.

\section{Methodology of multiple regression analysis}

Multiple regression analysis is the statistical process for estimating the relationships among the variables that have reason and result relation. The main focus of univariate regression is to analyze the relationship between a dependent variable and one independent variable and formulates the linear relation equation between dependent and independent variable (Whitcomb, 2012). The analysis of the relationships is hypothesized, and calculations of the parameters are used to develop a regression equation. Furthermore, various tests are employed to identify if the variables and the estimated model's adequacy. If the model is substantiated sufficient, the regression equation is used to predict the value of the dependent variable based on the values of the independent variables (Zaid, 2015). For this research, the IBM SPSS Software is chosen to compose a model. Moreover, standard multiple regression is selected as the type of analysis model in SPSS, because it includes predictors into the equation simultaneously. In accordance with Pallant (2010), simultaneously means "each independent variable is evaluated in terms of its predictive power, over and above that offered by all other independent variables". Furthermore, forced entry is selected as the method of conducting samples in the program.

Variables of the Quantitative Analysis. Multiple regression analysis includes two types of variables: dependent and several independent variables (collected from Bitcoin, 2018). The dependent variable is being tested and measured by using regressors. Independent variable is controlled in the analysis to test the effects on the dependent variable. Particularly, this multiple linear regression analysis includes one dependent variable (USD/EUR exchanges rate) (collected fro Bank of Lithuania, 2018) and 8 regressors. All indicators included are continuous, which is a quantity that has a changing value (has an infinite number of possible values) (Whitcomb, 2012).

The Hypothesis of Quantitative Research. The main aspiration is to identify how predictors will influence the dependent variable (Uriel, 2013). In accordance, how much the variance in the exchange rates of USD/EUR can be explained by the predictors.

$H_{0}:$ cryptocurrencies do not have the influence on the exchange rates USD/EUR.

$H_{A}$ : cryptocurrencies have the possible influence on the exchange rates USD/EUR.

The Assumptions of the Multiple Regression Analysis. In accordance with the literature, assumptions tested for this method are as follows (Čekanavičius \& Murauskas, 2014; Pallant, 2010; Whitcomb, 2012):

$I^{s t}$ Assumption. Linear in parameters; the stochastic process, where $x_{t 1}, x_{t 2}, \ldots, x_{t k}, y_{t}: t=1,2, \ldots, \mathrm{n}$ follows the linear model:

$$
Y_{t}=\beta_{0}+\beta_{1} x_{t 1}+\ldots \beta_{k} x_{t k}
$$

where $n$ is the number of observations (time periods); $t$ denotes the time period; $j$ is a label to indicate one of the $k$ explanatory variables; $Y_{t}$ is the dependent variable (explained variable); $x_{t j}$ are the independent variables (predictors).

$2^{\text {nd }}$ Assumption. Correlations. Firstly, in the underlying time series process, none of the independent variables has a perfect linear combination with the others. Secondly, the dependent variable and regressors should be highly correlated, however, the relationship between predictors must have weak correlation coefficient.

$3^{\text {rd }}$ Assumption. Homoscedasticity. This means that the residuals at each level of the predictors should have the same variance. However, in accordance with Whitcomb (2012), the estimates with heteroscedasticity are still valid in large time-series data but not optimal.

$4^{\text {th }}$ Assumption. Normally distributed errors: it assumes that the residuals in the model are normally distributed with the mean of 0 . The difference between the model and observed data are most frequently zero or very close to zero. 
$5^{\text {th }}$ Assumption. No multicollinearity: predictors of the model are not highly correlated with each other. If the analysis includes more than one independent variable, it could cause the concern of multicollinearity, when there is a strong correlation between two or more predictors.

However, assumptions are compounded in a general way, consequently, some of them can be ignored or modified depending on the type of the analysis, data and time interval. Each assumption is tested and confirmed, rejected, or violated by using different measurements: descriptive statistics, assumption and hypothesis testing (model summary table, correlation matrix, ANOVA, coefficients table $\left(\mathrm{VIF}^{2}\right)$ ), removing inacceptable variables (at each analysis stage), qualification of the equation (when analysis meets all assumptions after removing invalid variables), and result interpretation (Sheather, 2009).

\section{Multiple regression analysis of Bitcoin influence}

Analysis helps to indicate how well the group of selected variables are able to predict the stress levels and how much unique variance each of the predictors explains in the dependent variable (exchange rates USD/EUR), over and above other regressors (Sheather, 2009).

Descriptive Statistics. First of all, variables are presented mathematically by involving mean, standard deviation and number used in the model. Results are in Table 4 as per below.

Table 4. Descriptive statistic of variables used in the model (created by an author: SPSS output)

\begin{tabular}{|l|c|c|c|}
\hline \multicolumn{5}{|c|}{ DESCRIPTIVE STATISTICS } & N \\
\hline & Mean & Std. Deviation & 2647 \\
\hline USD/EUR & 1.21 & 0.11 & 2647 \\
\hline Cap_BTCU & 18084756113.79 & 34889770581.69 & 2647 \\
\hline MS_BTC & 12537976.62 & 3354877.22 & 2647 \\
\hline Price_BTCU & 1118.44 & 2667.46 & 2647 \\
\hline Transac_Fess_BTCU & 67.79 & 121.39 & 2647 \\
\hline Infl_rate_BTCU & 29.86 & 38.20 & \\
\hline
\end{tabular}

\subsection{The output of the Regression Analysis (SPSS)}

Five regressors were included in the model whereas these variables are considered to have the influence for the conventional banking industry. Analysis showed that not all variables are applicable in the model. The reliable analysis is created by reducing one at the stage least significant variable (or variable that could cause multicollinearity) from the model in accordance with R Squared and Pearson's correlation table (Yan \& Su, 2009). Overall, 4 analysis outputs were conducted until all assumptions of the analysis were satisfied. Variables as Price of Bitcoin in USD; Transaction fee of BTC expressed in USD; Market capitalization of BTC in USD was omitted from the model since they do not meet required assumptions. After all, only two independent variables are included in the final model equation as described below.

Final output. Now analysis is composed of 2 regressors: money supply and an inflation rate of BTC. As model summary Table 5 provides R Squared is quite high and equals to 0.595 . It specifies that even $59.5 \%$ of the total variance in the USD/EUR exchange rates is explained by the money supply and the inflation rate of the BTC. Adjusted R Square shows corrected value of the of $\mathrm{R}$ Square to prescribe better estimates for the true data set value. The data of this research has a large extent, R Square and Adjusted R Square are the same.

Table 5. Model fit (4) (created by an author: SPSS output)

\begin{tabular}{|c|c|c|c|c|}
\hline \multicolumn{4}{|c|}{ MODEL SUMMARY } \\
\hline Model & $\mathrm{R}$ & R Square & Adjusted R Square & Std. Error of the Estimate \\
\hline 1 & $0.771 \mathrm{a}$ & 0.595 & 0.595 & 0.073178857928871 \\
\hline \multicolumn{4}{l}{} \\
\hline \multicolumn{2}{l}{ a. Predictors: (Constant), Infl_rate_BTCU, MS_BTC } \\
\hline
\end{tabular}

Correlation matrix (Table 6) points out that the relationship between the dependent variable and regressors is not very high but sufficient (more than 0.3 ) to run the model.

${ }^{2}$ VIF - Variance Inflation Factor. 

of selected fiat currencies

Table 6. Table of correlations (4) (created by an author: SPSS output)

\begin{tabular}{|c|l|c|c|c|}
\hline \multicolumn{5}{|c|}{ CORRELATIONS } \\
\hline \multirow{3}{*}{ Pearson Correlation } & USD/EUR & MS_BTC & Infl_rate_BTCU \\
\hline & USD/EUR & 1.000 & -0.768 & 0.694 \\
\cline { 2 - 5 } & MS_BTC & -0.768 & 1.000 & -0.556 \\
\cline { 2 - 5 } & Infl_rate_BTCU & 0.694 & -0.556 & 1.000 \\
\hline \multirow{3}{*}{ Sig. (1-tailed) } & USD/EUR &. & 0.000 & 0.000 \\
\cline { 2 - 5 } & MS_BTC & 0.000 &. & 0.000 \\
\cline { 2 - 5 } & Infl_rate_BTCU & 0.000 & 0.000 & 2647 \\
\hline \multirow{3}{*}{ N } & USD/EUR & 2647 & 2647 & 2647 \\
\cline { 2 - 5 } & MS_BTC & 2647 & 2647 & 2647 \\
\cline { 2 - 5 } & Infl_rate_BTCU & 2647 & 2647 & \\
\hline
\end{tabular}

The money supply of BTC correlates with USD/EUR by Pearson's coefficient of -0.768 ; an inflation rate of BTC has a correlation index of $r=0.694$. Moreover, both regressors are not highly correlated with each other. This condition meets the requirement of no multicollinearity between independent variables.

Table 7 prescribes results of the ANOVA test. The null hypothesis states that all $\beta_{j}$ are equal to zero and there is no statistical significance in the model the alternative hypothesis states by contraries. Mathematically hypotheses are recorded as follow:

$$
\begin{aligned}
& H_{0}: \beta_{1}=\beta_{2}=\ldots=\beta_{n}=0 \\
& H_{A}: \beta_{j} \neq 0 ; j=1,2, \ldots, n .
\end{aligned}
$$

The indexes of a test refer that the model has linear parameters whereas ANOVA indicates statistical significance between coefficients of $\beta$ (p-value is less than $0.05(\mathrm{Sig} .=0.000)$ ). The null hypothesis is rejected, and the assumption of the inear relationship is met. As a result, further analysis is conducted.

Table 7. ANOVA test for significance (4) (created by an author: SPSS output)

\begin{tabular}{|c|c|c|c|c|c|c|}
\hline \multicolumn{7}{|c|}{ ANOVA } \\
\hline \multirow{2}{*}{ Model } & Sum of Squares & df & Mean Square & F & Sig. \\
\hline \multirow{2}{*}{1} & Regression & 20.806 & 2 & 10.403 & 1942.629 & $0.000^{\mathrm{b}}$ \\
\cline { 2 - 8 } & Residual & 14.159 & 2644 & 0.005 & & \\
\cline { 2 - 8 } & Total & 34.965 & 2646 & & & \\
\hline
\end{tabular}

a. Dependent Variable: USD/EUR

b. Predictors: (Constant), Infl_rate_BTCU, MS_BTC

Furthermore, Table 8 provides variance inflation for multicollinearity checking, tolerance index, and factor as the unique contribution of every regressor in explaining the variance of the dependent variable. The tolerance index in the table is high for both variable with the VIF lower than 5 (money supply and inflation rate's VIF $=3.738$ ). This condition confirms that the model of the analysis does not include multicollinearity between explanatory. The assumption of the no multicollinearity is fulfilled.

\begin{tabular}{|c|c|c|c|c|c|c|c|c|c|}
\hline \multicolumn{10}{|c|}{ COEFFICIENTS } \\
\hline & \multicolumn{2}{|c|}{$\begin{array}{l}\text { Unstandardized } \\
\text { Coefficients }\end{array}$} & \multirow{2}{*}{$\begin{array}{c}\begin{array}{c}\text { Standardized } \\
\text { Coefficients }\end{array} \\
\text { Beta }\end{array}$} & \multirow{2}{*}{$\mathrm{t}$} & \multirow{2}{*}{ Sig. } & \multicolumn{2}{|c|}{$\begin{array}{l}95.0 \% \text { Confidence } \\
\text { Interval for B }\end{array}$} & \multicolumn{2}{|c|}{$\begin{array}{l}\text { Collinearity } \\
\text { Statistics }\end{array}$} \\
\hline & B & Std. Error & & & & $\begin{array}{l}\text { Lower } \\
\text { Bound }\end{array}$ & $\begin{array}{l}\text { Upper } \\
\text { Bound }\end{array}$ & Tolerance & VIF \\
\hline (Constant) & 1.473 & 0.012 & & 120.222 & 0.000 & 1.449 & 1.497 & & \\
\hline MS_BTC & $-2.235 \mathrm{E}-8$ & 0.000 & -0.652 & -27.266 & 0.000 & 0.000 & 0.000 & 0.268 & 3.738 \\
\hline Infl_rate_BTCU & 0.000 & 0.000 & 0.135 & 5.656 & 0.000 & 0.000 & 0.001 & 0.268 & 3.738 \\
\hline
\end{tabular}

Table 8 . Table of coefficients (4) (created by an author: SPSS output) 

of selected fiat currencies

Finally, all assumptions of the regression analysis are met, and the model is described as certain, trustworthy, and reliable. Moreover, analyzed measures indicate, that there is a statistical significance in the model. Accordingly, the null hypothesis of the model is rejected: as the exchange rates of USD/EUR represents currency markets, the influence of cryptocurrencies to the conventional financial sector is very slight.

$\beta$ coefficients can be used to the composed conclusive equation of the model. Therefore, $\beta$ indexes impart how much each independent variable has a unique contribution in explaining the dependent variable. It shows the strength and direction of regressors influence. The table provides that the highest standardized $\beta$ coefficient belongs to the BTC money supply, which is -0.652 (Sig. $=0.000$ ) and has a negative relation. It has the strongest influence of the variation in explaining the dependent variable while all other variables are controlled. The inflation rate of BTC has a $\beta$ value of 0.135 , which is much lower. Nevertheless, the inflation rate's unique contribution of the variance in the dependent variable is still very important and has a statistical significance (Sig. $=0.000)$.

Since the model is accepted, the equation of the analysis can be concluded. Linear regression follows the data set consisting of measurements of the values of two $x$ and $Y$, to compose the model that is useful for predicting the value of the explained variable $Y$ for given values of $x$. The equation consists of:

$Y$ - the value of the explained variable;

$x$ - the value of regressors; predicting or explaining the value of $Y$;

$\beta_{0}$ - an intercept of the equation (constant); equals the value of $Y$ when $x_{j}=0$;

$\beta_{j}-$ standardized beta coefficient of $x$; the slope of the regression line. Shows how much $Y$ value varies for each one-unit change of $x$.

The equation of the research model to check the influence for exchange rates USD/EUR, which stands for conventional banking, by using regressors related to factors of cryptocurrencies, is as follow:

Whereas:

$$
Y=1.473-0.652 x_{1}+0.135 x_{2} .
$$

- An intercept, in accordance with the coefficients table of SPSS output, is $\beta_{0}=1.473$, which indicates the value of the dependent variable in the model, when all regressors (money supply and ian nflation rate of BTC) are equal to 0 .

$-\beta_{1}$ cthe oefficient of BTC money supply $\left(x_{1}\right)$ is equal to -0.652 and shows by how much USD/EUR varies in the model when $x_{1}$ changes by one unit.

$-\beta_{2}$ cthe oefficient of BTC inflation rate $\left(x_{2}\right)$ is equal to 0.135 and shows by how much USD/EUR varies in the model when $x_{1}$ changes by one USD.

Moreover, the correlation matrix and coefficients table impart that money supply of Bitcoins has a negative relationship with the exchange rates. It means that if the BTC supply in the market increases, the USD/EUR decreases by a certain unit. Moreover, the inflation rate has a positive relationship with exchange rates, hence, when the inflation rate of Bitcoins increases, USD/EUR increases as well.

\subsection{Result consideration of regression analysis}

After conducting the model of regression analysis with four different outcomes, the null hypothesis is rejected, and it is identified that the influence of cryptocurrencies, based on actual historical data, is very slight and could be assumed as negligible. By considering all factors included in the research, only money supply and an inflation rate of cryptocurrencies have the influence for conventional banking. Market capitalization, transaction fees, the price of Bitcoin were indicated as not statistically significant in the model, consequently, they were omitted.

The analysis imparts a negative relation between money supply and exchange rates. It means that if the number of BTC in the circulation of the market increases, exchange rates of USD/EUR should decrease. Moreover, positive relation occurs between inflation rate and USD/EUR. It means that if the inflation rate of BTC decreases, the value of exchange rates USD/EUR rises. However, in accordance with the analysis, the influence started to increase from 2011.

\section{Conclusions}

In accordance with analyzed literature, digital money brings economic (no intermediation, new opportunities for enterprises) and individual (personal data security) benefits. However, cryptocurrencies have some limitations: anonymity encourages to use virtual money for illegal purchases, "Black market", money laundering, etc. This risk could be emphasized as one of the most harmful for the economy. Moreover, based on theoretical analysis, there are several groups of researchers with different attitudes: (1) who believe that digital money and the Blockchain will alter payments; (2) who suppose that Bitcoin is one more economic bubble; (3) and those, who supports Blockchain and counts digital money as an asset.

Furthermore, as a matter of fact, findings of quantitative research prescribe that the influence of bitcoins, based on actual historical data, is very slight and negligible. It can be assumed that if the number of BTC in the circulation 

of selected fiat currencies

of the market increases, exchange rates of USD/EUR should slightly decrease. Moreover, if the inflation rate of BTC drops, the exchange rates of USD/EUR should slightly rise. Moreover, it is asserted the influence of digital money initiated to increase from 2011, when Bitcoin started to gain popularity and be traded to a greater extent. The current influence of bitcoins is too weak to make any significant affection for the fiat currency market.

To finalize, digital money can be expected to become the mean of exchange with a decentralized payment system. However, it might not be already existing cryptocurrencies since current influence is weak. New digital money could occur with more acceptable properties for payments. In addition, new currency should be based on the improved decentralized networking, whereas currently Blockchain is in its initial stage and is not able to cover a huge extent of transactions. However, banks can embrace Blockchain and digital money to preserve the bank's importance, improve transparency, and cost-effectiveness. Also, they might use Blockchain to maintain a close relationships with fintech enterprises, thus promoting economic growth and accelerating the development of new technologies. Likewise, the public should be more acquainted with the cryptocurrencies, Blockchain, its applicability, and opportunities.

\section{References}

Antonopoulos, A. M. (2014). Mastering Bitcoin: unlocking digital cryptocurrencies (1 ed.). New York: O’Reilly Media, Inc. Bitcoin. (2018). Bitcoin charts. Retrieved from https://charts.bitcoin.com/chart

Bollen, R. (2013). The legal status of online currencies: are Bitcoins the future? Journal of Banking and Finance Law and Practice, 24(3), 272-293.

Čekanavičius, V., \& Murauskas, G. (2014). Taikomoji regresinè analizè socialiniuose tyrimuose [Applied regression analysis in social research]. Vilnius: Vilniaus universiteto leidykla.

European Banking Authority. (2014, July 4). EBA opinion on 'virtual currencies'. Retrieved from https://eba.europa.eu/documents/10180/657547/EBA-Op-2014-08+Opinion+on+Virtual+Currencies.pdf

Bank of Lithuania. (2018). European Central Bank exchange rates. Retrieved from https://www.lb.lt/en/currency?currency $=$ USD\&class $=$ Eu\&type $=$ day\&date_from_day=2018-03-27\&date_to_day=2018-04-27

Glaser, F., Kai, Z., Haferkorn, M., Weber, M. C., \& Siering, M. (2014). Bitcoin-asset or currency? Revealing users' hidden intentions: complete research. In Twenty Second European Conference on Information Systems (pp. 1-14). Tel Aviv.

Hileman, G., \& Rauchs, M. (2017). Global cryptocurrency benchmarking study, University of Cambridge; Judge Business School. Marmelab. https://doi.org/10.2139/ssrn.2965436

Houben, R., \& Snyers, A. (2018). Cryptocurrencies and Blockchain: legal context and implications for financial crime, money laundering and tax evasion. Retrieved from http://www.europarl.europa.eu/cmsdata/150761/TAX3\%20Study\%20on\%20cryptocurrencies\%20and\%20blockchain.pdf

Huillet, M. (2018). Bitcoin is "Epidemic of enthusiasm" says Nobel prize winner Robert Shiller. Retrieved from https://cointelegraph.com/news/bitcoin-is-epidemic-of-enthusiasm-says-nobel-prize-winner-robert-shiller

Khvan, A. (2016). A regression analysis of cryptocurrency influence on the US dollar. Moscow: The Economic Faculty of Lomonosov Moscow State University. https://doi.org/10.2139/ssrn.2843142

Luther, W. J., \& White, L. H. (2014). Can Bitcoin become a major currency? (Working Paper No. 14-17). George Mason University, Department of Economics.Nair, G. R., \& Sebastian, S. (2017). Blockchain technology: Centralised ledger to distributed ledger. International Research Journal of Engineering and Technology, 4(3), 2823-2827.

Nakamoto, S. (2009). Bitcoin: a peer-to-peer electronic cash system. Bitcoin. Retrieved from https://bitcoin.org/bitcoin.pdf

Narayanan, A., Bonneau, J., Felten, E., Miller, A., \& Goldfeder, S. (2016). Bitcoin and cryptocurrency technologies: a comprehensive introduction. United Kingdom: Princeton University Press.

Reid, D. (2018). Bitcoin savaged by BIS as 'a bubble, Ponzi scheme and an environmental disaster'. Retrieved from https://www.cnbc.com/2018/02/06/bitcoin-and-cryptocurrencies-are-ponzi-bubbles-says-bis.html

Rosic, A. (2016). What is cryptocurrency: everything you need to know. BlockGeeks. Retrieved from https://blockgeeks.com/guides/what-is-cryptocurrency/

Sheather, S. (2009). A modern approach to regression with R. New York: Springer. https://doi.org/10.1007/978-0-387-09608-7

Tapscott, D., \& Tapscott, A. (2016). Blockchain revolution: how the technology behind Bitcoin is changing money, business, and the world. Penguin.

Uriel, E. (2013). Hypothesis testing in the multiple regression model. Universidad de Valencia. Retrieved from https://www.uv.es/uriel/4\%20Hypothesis\%20testing\%20in\%20the\%20multiple\%20regression\%20model.pdf

Vejačka, M. (2014). Basic aspects of cryptocurrencies. Journal of Economy, Business and Financing, 2, 75-83.

Wearden, G. (2018). Bitcoin won't last in world of finance, warns Nobel-winning economist. The Guardian. Retrieved from https://www.theguardian.com/business/2018/jan/25/bitcoin-wont-last-in-world-of-finance-warns-nobel-winning-economist

Whitcomb, K. (2012). Study guide to accompany statistical techniques business \& economics (15 $5^{\text {th }}$ ed.). New York: MCGrawHill/Irwin.

Yan, X., \& Su, X. (2009). Linear regression analysis: theory and computing. Singapore: World Scientific Publishing Co. Pte. Ltd. https://doi.org/10.1142/6986

Zaid, M. A. (2015). Correlation and regression analysis TEXTBOOK. Ankara: SESRIC.

Zaninotto, F. (2016). The Blockchain explained to web developers, Part 1: the theory. Marmelab. Retrieved from https://marmelab.com/blog/2016/04/28/blockchain-for-web-developers-the-theory.html

Zhao, Y. (2015). Cryptocurrency brings new battles into the currency market. In Seminar Future Internet WS2014. (pp. 91-99). Germany: Technische Universität München. 